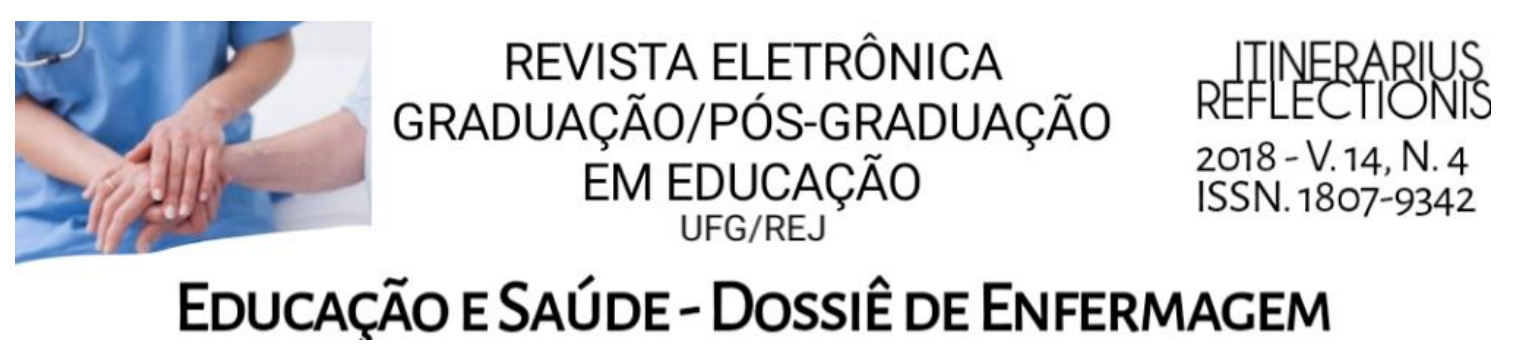

\title{
CUIDADORAS DE IDOSOS E AS RELAÇÕES ESTABELECIDAS NO ATO DE CUIDAR
}

\author{
Gabriela Jorge de Novaes ${ }^{1}$ \\ Luana de Moraes ${ }^{2}$ \\ Lara Thaiane Souza Pereira ${ }^{3}$ \\ Marise Ramos de Souza ${ }^{4}$ \\ Cristiane José Borges ${ }^{5}$
}

\begin{abstract}
RESUMO: A expectativa de vida da população tem aumentado significativamente. E concomitantemente ao envelhecimento populacional emerge a figura do cuidador de idoso, com um papel fundamental no processo de cuidar da pessoa com idade igual ou superior a 60 anos. Nessa perspectiva, o presente estudo objetivou identificar quais são as relações construídas entre as mulheres cuidadoras e os idosos. Trata-se de uma pesquisa descritiva, de abordagem qualitativa. Foram entrevistadas nove mulheres cuidadoras, com média de idade de 44,7 anos, sendo em sua maioria separadas, evangélicas, com renda familiar entre 1 a 2 salários mínimos, com vínculo formal e informal e que cuidam apenas de um idoso. Os dados revelaram que ao dispensar o cuidado ao idoso com alguma limitação funcional é inevitável o estabelecimento de vínculo afetivo entre a mulher cuidadora e a pessoa idosa, demonstrando assim, a satisfação no ato de cuidar. O estudo evidenciou que os aspectos positivos em relação à assistência prestada ao idoso, auxilia na desmistificação da existência apenas de consequências negativas nesse tipo de cuidado.
\end{abstract}

Palavras chaves: Idoso; Cuidador; Enfermagem.

\section{ELDERLY CAREGIVERS AND RELATIONS ESTABLISHED IN THE ACT OF CARE}

ABSTRACT: The population life expectancy has increased significantly. Concomitant to the aging population we see to emerge the elderly caregiver figure with a fundamental role in the caring process for person aged 60 or over. From this perspective, the present study aimed to identify the relationships built between women caregivers and the elderly. This is a descriptive, qualitative approach. Nine female caregivers were interviewed, with a mean age of 44.7 years, being mostly separated, evangelicals, with family income between 1 and 2 minimum wages, with formal and informal bonding and who only care for one elderly person. The data revealed that when dispensing care for the elderly with some functional limitation, it is inevitable to establish an affective bond between the caregiver and the elderly person, thus, demonstrating satisfaction in the act of caring. The study showed that the positive

\footnotetext{
${ }^{1}$ Enfermeira. Egressa do Programa de Educação Tutorial - PET Enfermagem UFG/Regional Jataí. Email: bi.jorge@yahoo.com.br

2 Enfermeira. Egressa do Programa de Educação Tutorial - PET Enfermagem UFG/Regional Jataí. Email: luhmrs08@hotmail.com

${ }^{3}$ Enfermeira. Egressa do Programa de Educação Tutorial - PET Enfermagem UFG/Regional Jataí. Email: biomedlara@gmail.com

4 Enfermeira. Professora doutora do Curso de Graduação em Enfermagem. Tutora do Programa de Educação Tutorial - PET Enfermagem UFG/Regional Jataí. Email: msc_marise@hotmail.com

5 Enfermeira. Professora doutora do Curso de Graduação em Enfermagem. Tutora do Programa de Educação Tutorial - PET Enfermagem UFG/Regional Jataí. Email: cristianejose@yahoo.com.br
} 


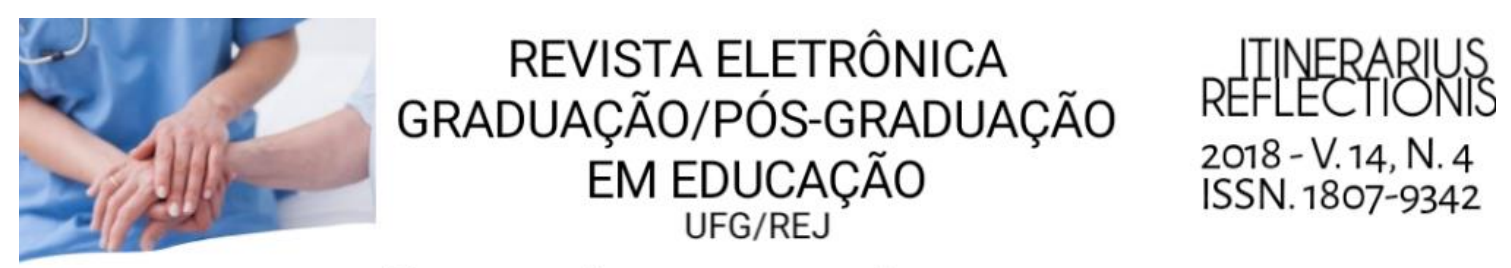

\section{EdUCAC̨Ãoe SAÚdE-DOSSIÊ DE ENFERMAGEM}

doença crônica pode favorecer para o desenvolvimento de habilidades pessoais e técnicas, o que consequentemente contribui para prestação de assistência com amor e carinho. Neste contexto, surge a indagação: como são constituídas as relações entre as mulheres cuidadoras e os idosos?

O presente artigo teve como objetivo avaliar as relações constituídas entre as mulheres cuidadoras e os idosos, durante o processo de cuidar.

\section{MÉTODO}

Trata-se de uma pesquisa descritiva, de abordagem qualitativa, realizada com mulheres cuidadoras de idosos em um município localizado na região sudoeste, do estado de Goiás.

Para a seleção da amostra utilizou-se os dados cadastrais de cuidadores de idosos que estiveram presentes no I e/ou II Curso Básico para Cuidadores de Idosos, nos anos de 2013 e 2014, organizado pelo Grupo do Programa de Educação Tutorial (PET) do curso de Enfermagem da Universidade Federal de Goiás (UFG) Regional Jataí. Este grupo está em atividade desde o ano de 2010 e promove ações de ensino, pesquisa, e extensão voltadas tanto para comunidade universitária quanto externa.

A partir dos dados cadastrais, as mulheres cuidadores foram convidadas por meio de contato telefônico a fazerem parte do estudo e aquelas que aceitaram, foram contactadas em um segundo momento, num em um encontro previamente agendado, para assinatura do termo de consentimento livre e esclarecido e realização da entrevista.

A amostra foi constituída por nove participantes, que atenderam os seguintes critérios de inclusão: atuar como cuidadora formal ou informal de idosos; possuir idade igual ou superior a dezoito (18) anos; ser do sexo feminino, ter participado do | e/ou I| Curso Básico para Cuidadores de Idosos promovido pelo Grupo do PET Enfermagem - UFG- Regional Jataí e ser cuidadora há no mínimo 4 meses. Foram excluídas todas as cuidadoras que não possuíam disponibilidade de horário para a realização da entrevista.

O número de participantes foi determinado pelo critério de saturação dos dados, o que segundo Minayo (2014) é quando o pesquisador adquiri o 


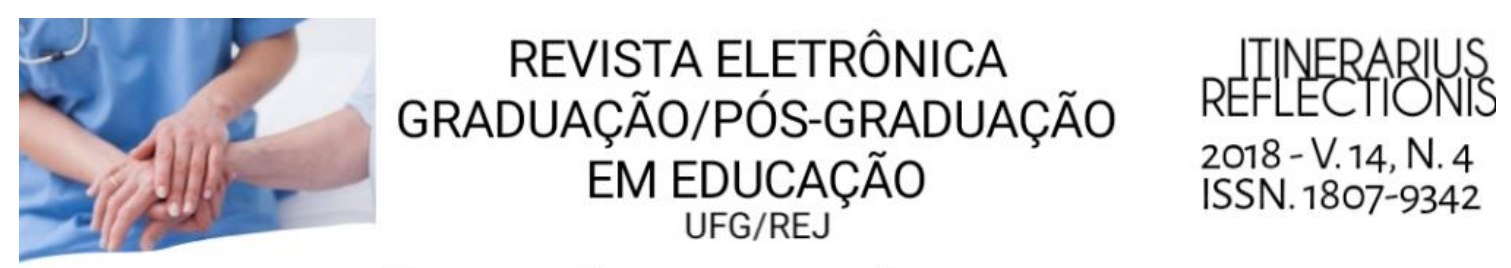

\section{EdUCAÇÃo ESAÚdE-DOSSIÊ DE ENFERMAGEM}

conhecimento das homogeneidades, da diversidade e da intensidade das informações indispensáveis para o desenvolvimento do estudo.

A entrevista individual e semiestruturada aconteceu após um consenso entre a disponibilidade de data e horário entre as pesquisadoras e as mulheres cuidadoras. A mesma foi realizada na residência de cada participante.

A coleta de dados foi efetivada no período compreendido entre os meses de abril e maio de 2015. O instrumento de coleta foi composto por duas etapas, sendo a primeira constituída por dados sociodemográficos (faixa etária, estado civil, escolaridade, renda familiar) e o vínculo com o idoso. E a segunda pela questão norteadora: Como é a sua relação com o idoso que você cuida?

A mesma foi gravada, tendo a duração média de 55 minutos. A entrevista foi transcrita na íntegra, no entanto, devido alguns erros de linguagem que dificultaria a compreensão do leitor foram parcialmente editadas. Para manter o anonimato dos participantes do estudo, estas foram identificadas com a utilização de codinomes "C1, C2, ..."

Os dados foram organizados e analisados de acordo com as etapas preconizadas Bardin (2011), ou seja, pré-análise, exploração do material e tratamento dos resultados, inferência e interpretação. A partir da análise emergiram três categorias temáticas, sendo elas: a dependência do idoso, o ato de cuidar e o estabelecimento de laços afetivos e por último, a satisfação no ato de cuidar do idoso.

O projeto foi aprovado pelo Comitê de Ética e Pesquisa do Hospital das Clinicas de Goiás da Universidade Federal de Goiás, sob 0 ํo- protocolo 34077014.1.0000.5078, atendendo aos aspectos éticos de pesquisa envolvendo seres humanos, conforme Resolução no 466 de 12 de dezembro de 2012 (BRASIL, 2012).

\section{RESULTADOS E DISCUSSÃO}

\section{Caracterizando as mulheres cuidadoras de idosos}

Participaram do estudo mulheres cuidadoras de idosos com faixa etária entre 34 a 62 anos, sendo a média de idade de 44,7 anos. Das nove participantes do estudo quatro $(44,4 \%)$ referiram ser separadas, seguido de três $(33,3 \%)$ solteiras e 


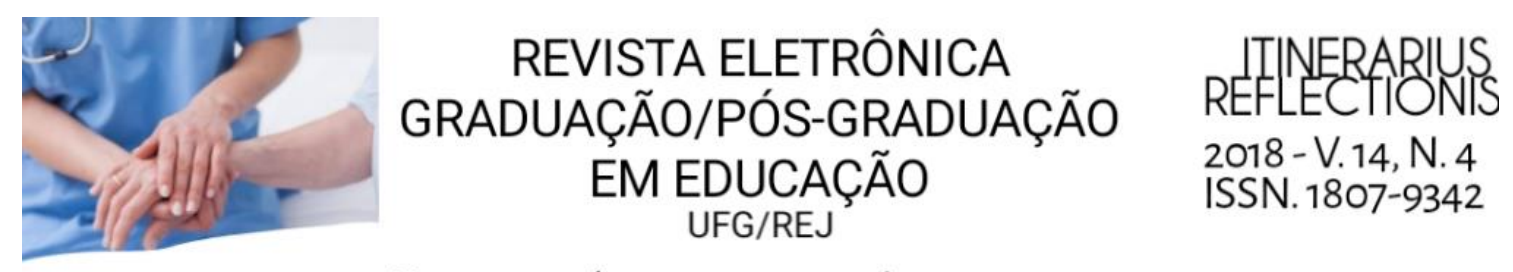

\section{EduCAÇÃo ESAÚdE-DoSSIÊ DE ENFERMAGEM}

[...] Com idosos eu tenho o maior carinho [...] talvez seja um dom meu (C8).

$\mathrm{Na}$ literatura observou-se que geralmente, são os cuidadores informais que relacionam a assistência ao idoso como retribuição pela atenção recebida em momentos anteriores de suas vidas e sentem-se satisfeitos por serem capazes desempenharem essa atividade (OLIVEIRA; CALDANA, 2012), porém para alguns cuidadores o papel pode ser visto com uma obrigação (ALMEIDA; JARDIM; FRANCO, 2014) e esse sentimento reflete consideravelmente na maneira de prestar o cuidado (AREOSA et al., 2014).

Nesse sentido, o sentimento de satisfação por vezes é substituído por sentimentos negativos, como raiva e impaciência, em situações críticas que exigem maior envolvimento do cuidador (OLIVEIRA; CALDANA, 2012).

Os achados deste estudo destacam duas $(22,2 \%)$ cuidadoras que relataram satisfação frente ao ato de cuidar. Em estudo desenvolvido por Lindquist et al. (2012) observou-se que a maioria $(65,3 \%)$ dos cuidadores formais relataram a profissão como sendo gratificante, devido ao aprendizado que adquirem com o idoso, e a segunda razão por optarem pelo trabalho de cuidador é a remuneração, sendo considerado como um aspecto motivador. Esses profissionais afirmaram escolher a ocupação principalmente por afinidade e por necessidades financeiras.

No estudo de Garbin et al. (2010) além dos fatores supracitados, os cuidadores relataram assumir essa função inesperadamente e a respeito dessa ocorrência os autores enfatizaram a necessidade de qualificação do indivíduo para executar suas atividades e fornecer o cuidado adequado ao idoso.

Areosa et al. (2014) apontam diferenças no discurso de cuidadores sobre a relação de sentimentos no cuidar, os cuidadores formais elencam geralmente: pena, amor, adoração e afeto; enquanto os informais: obrigação, cansaço, esforço e stress. Essas divergências se estendem a outras condições, como o tempo de dedicação ao idoso e o motivo para o desempenho do cuidar.

Nos achados do presente estudo determinados relatos apresentaram semelhanças referentes aos sentimentos expressados pelos cuidadores no estudo supracitado. Foi descrito pelos cuidadores formais sentimentos positivos perante a função que desempenham: 


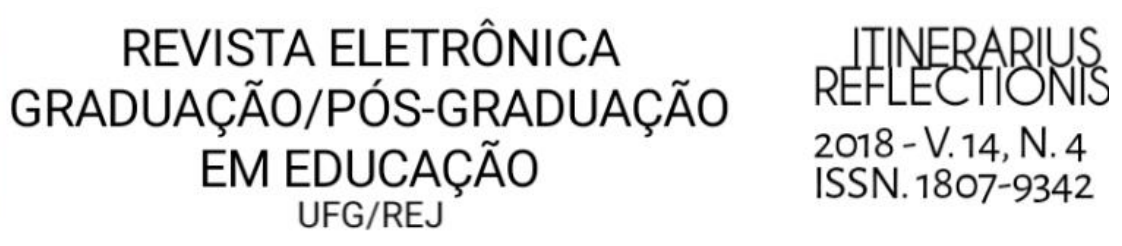

\section{EdUCAÇÃo ESAÚDE-DOSSIÊ DE ENFERMAGEM}

"E eu era [...] muito carinhosa porque eu amava ela [...]". (C8)

"Eu assim, eu sou carinhosa, eu faço meu trabalho com amor, faço porque eu gosto de fazer [...]". (C8)

“[...] Quando eu vejo paciente lá assim que ele tá bem [...] eu fico bem comigo mesma [...]". (C8)

"[...] Porque quer queria ou quer não, você se envolve, não tem como você não se envolver [...]". (C9)

Enquanto o relato de uma cuidadora informal expõe o estresse do cuidar:

"[...]Além de ter minha mãe para cuidar [...] ainda tem filho, tem a casa, [...] meu marido, tem tudo [...]. Então é meio desgastante [...] é por isso que eu fico meio assim cansada, [...] não tenho cansaço físico, [...] eu tenho mais é o emocional[...] cansa muito, dói a cabeça, dá nervoso, stress [...] mas é assim [...]". (C7)

Entretanto, o desgaste no cuidado, também revelado nos relatos de cuidadoras formais:

"[...] É porque eu estava trabalhando muito, eu estava trabalhando durante o dia e a noite. Eu morei no serviço também, então foi muito desgaste, sabe, como físico e mental $[\ldots] " .(\mathrm{C} 6)$

"[...] Além do desgaste físico que o cuidador tem, porque tem, aliás, como toda profissão [...] tem um desgaste físico, e quando você não tem o desgaste físico você tem mental. E o cuidador eu acho que ele sofre os dois, tanto físico quanto mental [...]". (C9)

O estudo revelou também que o tempo de dedicação ao idoso foi um diferencial entre os relatos de cuidadores formais e informais. Observou-se o relato de uma cuidadora informal mencionando a dificuldade em ser a única responsável pelos cuidados ao idoso: 


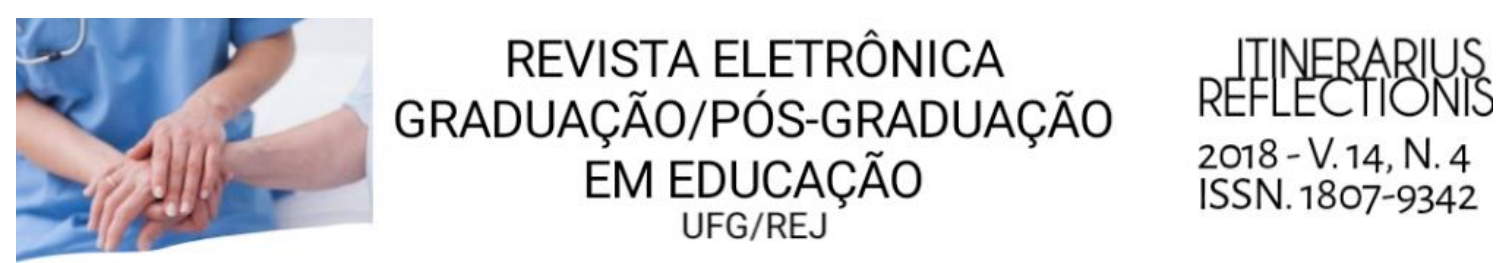

\section{EdUCAÇÃo ESAÚDE-DOSSIÊ DE ENFERMAGEM}

"[...] Ela depende de mim, né, porque só eu tenho que cuidar dela [...] eu tenho uma família pequena, né, porque assim eu não tenho outro irmão[...]". (C7).

Contudo, notou-se que a cuidadora formal, na maioria das vezes, compartilha os cuidados do idoso com os familiares do mesmo.

"[...] Os familiares todos ajudam, a família é grande, todos revezam e ajudam. E assim o elo é muito grande da família inteira com ele, sabe. E ninguém se desprende [...]." (C9).

Os dados do estudo revelaram que a atenção dispensada aos idosos é diferente do cuidado direcionado a outros indivíduos, à exemplo de crianças, como mencionado por uma das entrevistadas:

"Eu não tenho paciência com criança, de jeito nenhum, nem com meus netos, mas com idosos eu tenho o maior carinho assim, parece que, talvez seja um dom meu." (C8).

A assistência ao idoso consequentemente exige capacidades específicas para o cuidado qualificado e humanizado. Verifica-se nesse sentido, que as habilidades do cuidador em sua atuação interferem no resultado do cuidado prestado, visto que a ausência de preparo para a função pode favorecer ao desenvolvimento de danos ao idoso, que se encontra à mercê daquele que cuida, assim como pode acarretar em consequências prejudicais para o próprio cuidador, como a ansiedade e o esgotamento físico (MARQUES; TEIXEIRA; SOUZA, 2012).

\section{CONCLUSÃO}

O presente estudo evidenciou que as relações constituídas entre as mulheres cuidadoras e os idosos durante o processo de cuidar, envolvem diversos sentimentos positivos, entre eles o afeto, carinho e satisfação no ato de cuidar do idoso, desmistificando assim, a concepção de que o ato de cuidar da pessoa idosa está circundado somente por consequências negativas, como a sobrecarga e o sofrimento emocional daquele que executa o cuidado.

Embora esses sentimentos sejam significativos nas relações entre as mulheres cuidadoras e o idoso, é necessário também destacar os efeitos positivos 


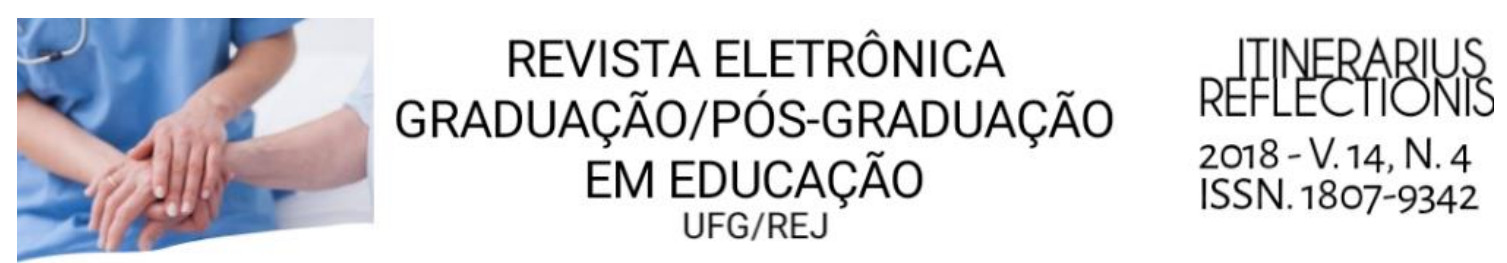

\section{EdUCAC̨Ãoe SAÚdE-DossIÊ DE ENFERMAGEM}

que podem ser estabelecidos na conexão entre estes indivíduos, o qual permiti determinar um forte vínculo afetivo, sendo este rompido somente após a morte, em geral, da pessoa cuidada.

Nesta perspectiva, considera-se que os profissionais da área da saúde ao dispensarem à atenção ao cuidador de idoso, em especial às mulheres, devem ressaltar e valorizar os aspectos positivos no ato de cuidar, minimizando assim, 0 enfoque somente para o desgaste físico e emocional do cuidador. Acredita-se que, ajudar estes indivíduos a terem um olhar por um outro prisma sobre o ato de cuidar da pessoa idosa poderá auxiliar no fortalecimento das relações entre os indivíduos envolvidos no processo, além de possibilitar uma maior qualidade na assistência prestada ao idoso.

\section{REFERÊNCIAS}

ALMEIDA, L.G.R.S.; JARDIM, M.G.; FRANCO, E.C.D. O cuidar do idoso com Alzheimer: sentimentos e experiências vivenciados por seus cuidadores. Revista de Enfermagem da UFSM, Santa Maria, v. 4, n.2, p. 303-312, 2014.

ALVES, J.M.P.M. O que eu faço tem valor: discutindo o cuidado familiar e o reconhecimento. Ex aequo, Lisboa, n. 30, p. 97-111, 2014.

ARAUJO, J.S. et al. Perfil dos cuidadores e as dificuldades enfrentadas no cuidado ao idoso, em Ananindeua, PA. Rev. Bras. Geriatr. Gerontol, Rio de Janeiro, v. 16, n. 1, p. 149-158, 2013.

AREOSA, S.V.C. et. al. Cuidar de si e do outro: estudo sobre os cuidadores de idosos. Psicologia, saúde \& doenças, Lisboa, v. 15, n.2, p. 482-494, 2014.

BARDIN, L. Análise de Conteúdo. São Paulo: Edições 70, 2011.

BAUAB, J.P.; EMMEL, M.L.G. Mudanças no cotidiano de cuidadores de idosos em processo demencial. Rev. Bras. Geriatr. Gerontol, Rio de Janeiro, v. 17, n. 2, p. 339-352, 2014.

BRASIL. Presidência da República. Lei № 8.842, de 4 de janeiro de 1994. Dispõe sobre a política nacional do idoso, cria o Conselho Nacional do Idoso e dá outras providências. Brasília, DF, 1994.

BRASIL. Presidência da República. Lei $n^{\circ} 10.741$, de 1ํ de outubro de 2003. Dispõe sobre o Estatuto do Idoso e dá outras providências. Brasília, DF, 2003.

BRASIL. Ministério da Saúde. Resolução no 466, de 12 de dezembro de 2012. Brasília, DF: Conselho Nacional de Saúde, 2012.

BRASIL, M.S. Guia Prático do Cuidador. Brasília: Ministério da Saúde, 2008. 


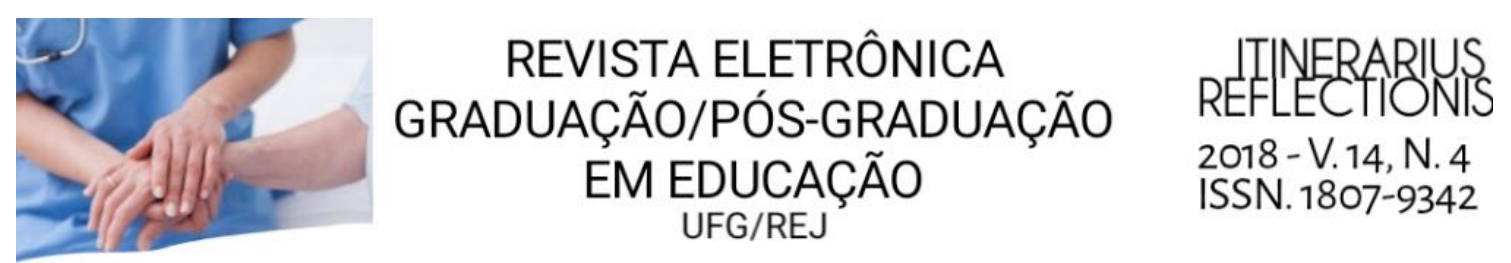

\section{EdUCAÇÃO E SAÚdE-DOSSIÊ DE ENFERMAGEM}

CAMARANO, A.M; KANSO, S; MELLO, J.L. Como vive o idoso brasileiro. In: CAMARANO, A.M (org). Novos idosos brasileiros muito além dos 60 ? Rio de Janeiro: Instituto de Pesquisa Econômica Aplicada, 2004. p.25-59.

COLOMÉ, I.C.S. et.al. Cuidar de idosos institucionalizados: características e dificuldades dos cuidadores. Rev. Eletr. Enf, Goiânia, v.13, n. 2, p. 306-12, 2011.

DEBERT, G.G.; OLIVEIRA, A.M. A profissionalização da atividade de cuidar de idosos no Brasil. Revista Brasileira de Ciência Política, Brasília, n.18, p. 7-41, 2015.

DE VALLE-ALONSO, M. J. et. al. Sobrecarga y Burnout en cuidadores informales del adulto mayor. Enfermería Universitaria, v.12, n.1, p. 19-27, 2014.

DEL DUCA, G.F.; MARTINEZ, A.D.; BASTOS, G.A.N. Perfil do idoso dependente de cuidado domiciliar em comunidades de baixo nível socioeconômico de Porto Alegre, Rio Grande do Sul. Ciênc. saúde coletiva, Rio de Janeiro, 17, n.5, p. 1159-1165, 2012.

ERVATTI, L. R.; BORGES, G. M.; JARDIM, A. P. (Org). Mudança demográfica no Brasil no início do século XXI: subsídios para as projeções da população. Rio de Janeiro: Instituto Brasileiro de Geografia e Estatística, 2015. P.156.

FRATEZI, F.R.; GUTIERREZ, B.A.O. Cuidador familiar do idoso em cuidados paliativos: o processo de morrer no domicílio. Ciência \& Saúde Coletiva, Rio de Janeiro, v. 16, n. 7, p. 3241-3248, 2011.

FUHRMANN, A.C.; BIERHALS, C.C.B.K.; SANTOS, N.O.; PASKULIN, L.M.G. Associação entre a capacidade funcional de idosos dependentes e a sobrecarga do cuidador familiar. Rev. Gaúcha Enferm, Porto Alegre, v. 36, n.1, p. 14-20, 2015.

GAIOLI, C.C.L.O.; FUREGATO, A.R.F.; SANTOS, J.L.F. Perfil de cuidadores de idosos com doença de Alzheimer associado à resiliência. Texto \& Contexto Enferm, Florianópolis, v. 21 n.1, p. 150-157, 2012.

GARBIN, C.A.S. et al. O envelhecimento na perspectiva do cuidador de idosos. Ciência \& Saúde Coletiva, Rio de Janeiro, v.15, n. 6, p. 2941-2948, 2010.

GONÇALVES, L.T.H.; LEITE, M.T.; HILDEBRANDT, L.M.; BISOGNO, S.C.; BIASUZ, S.; FALCADE, B.L. Convívio e cuidado familiar na quarta idade: qualidade de vida de idosos e seus cuidadores. Rev. Bras. Geriatr. Gerontol, Rio de Janeiro, v. 16, n.2, p. 315-325, 2013.

GRATÃO, A.C.M.; TALMELLI, L.F. da S.; FIGUEIREDO, L.C.; ROSSET, I.; FREITAS, C.P.; RODRIGUES, R.A.P. Dependência funcional de idosos e a sobrecarga do cuidador. Rev Esc Enferm USP, São Paulo, v. 47, n.1, p. 137-144, 2013. 


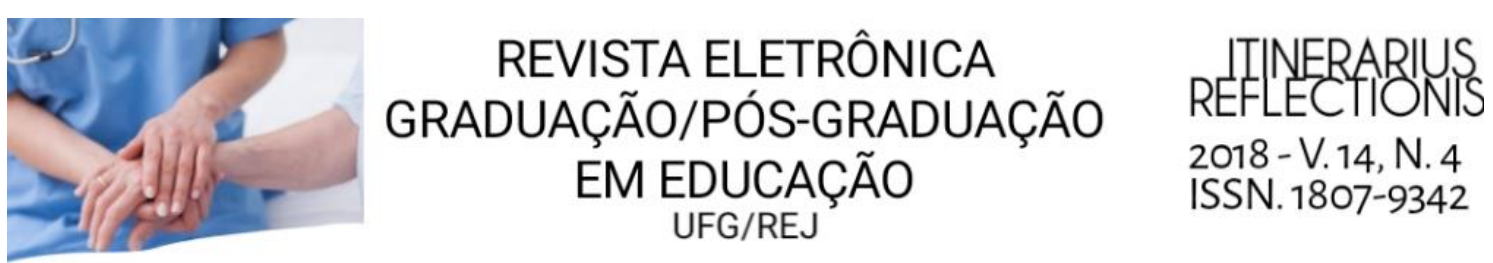

\section{EDUCAÇÃo ESAÚdE-DOSSIÊ DE ENFERMAGEM}

GUEDES, A.C.; PEREIRA, M. da G. Sobrecarga, enfrentamento, sintomas físicos e morbidade psicológica em cuidadores de familiares dependentes funcionais. Rev. Latino-Am. Enfermagem, v. 21, n. 4, p. 01-06, 2013.

GUIMARÃES M.L, et. al. O cuidado ao idoso em saúde coletiva: um desafio e um novo cenário de prática. In: SOUZA, M.C.M.R; HORTA, N.C (org). Enfermagem em saúde coletiva: teoria e prática. São Paulo: Guanabara Koogan, 2012.

IAVARONE, A. et. al. Caregiver burden and coping strategies in caregivers of patients with alzheimer's disease. Neuropsychiatric Disease and Treatment, v. 10, p. 1407-1413, 2014.

LENARDT, M.H.; WILLIG, M.H.; SEIMA, M.D.; PEREIRA, L. de F. A condição de saúde e satisfação com a vida do cuidador familiar de idoso com Alzheimer. Colomb Med, Colombia, v. 42, p.17-25, 2011.

LINDQUIST, L.A.; TAM, K.; FRIESEMA, E.; MARTIN, G.J. Paid caregiver motivation, work conditions, and falls among senior clients. Arch Gerontol Geriatr, v. 55, n. 2, p. 442-445, 2012.

LOUREIRO, L.S.N. et. al. Sobrecarga de cuidadores familiares de idosos: prevalência e associação com características do idoso e do cuidador. Rev Esc Enferm USP, São Paulo, v. 47, n. 5, p. 1133-1140, 2013.

MARQUES, M.J.F.; TEIXEIRA, H.J.C.; SOUZA, D.C.D.B.N. Cuidadoras informais de Portugal: vivências do cuidar de idosos. Trab. educ. saúde, Rio de Janeiro, v.10, n. 1, p. 147-159, 2012.

MAZZA, M.M.P.R.; LEFEVRE, F. Cuidar em família: análise da representação social da relação do cuidador familiar com o idoso. Rev. bras. crescimento desenvolv. Hum, São Paulo, v.15, n.1, p. 1-10, 2005.

MINAYO, M.C. de S. O desafio do conhecimento: pesquisa qualitativa em saúde. 14ํe․ São Paulo: Hucitec, 2014.

NARDI, E.F.R.; SAWADA, N.O.; SANTOS, J.L.F. Associação entre a incapacidade funcional do idoso e a sobrecarga do cuidador familiar. Rev. Latino-Am. Enfermagem, v.21, n. 5, p. 1096-1103, 2013.

OLIVEIRA, A.P.P.; CALDANA, R.H.L. As repercussões do cuidado na vida do cuidador familiar do idoso com demência de Alzheimer. Saúde Soc. São Paulo, São Paulo, v. 21, n.3, p. 675-685, 2012.

PEREIRA, D.S.; NOGUEIRA, J.A.D.; SILVA, C.A.B. Qualidade de vida e situação de saúde de idosos: um estudo de base populacional no Sertão Central do Ceará. Rev. Bras. Geriatr. Gerontol, Rio de Janeiro, v. 18, n.4, p. 893-908, 2015.

PIEXAK, D.R.; FREITAS, P.H.; BACKES, D.S.; MORESCHI, C.; FERREIRA, C.L.L.; SOUZA, M.H.T. de Percepção de profissionais de saúde em relação ao cuidado a 


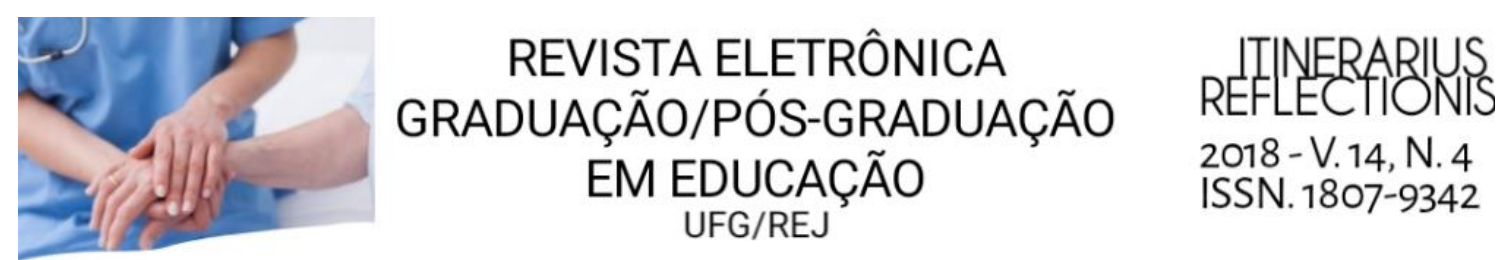

\section{EdUCAÇÃoe SAÚdE-DossIÊ DE ENFERMAGEM}

pessoas idosas institucionalizadas. Rev. Bras. Geriatr. Gerontol, Rio de Janeiro, v.15, n. 2, p. 201-208, 2012.

ROCHA, B.M.P.; PACHECO, J.E.P. Idoso em situação de dependência: estresse e copingdo cuidador informal. Acta Paul Enferm, São Paulo, v. 26, n.1, p. 50-6, 2013.

SANTOS, A.A. dos.; PAVARINI, S.C.I. Perfil dos cuidadores de idosos com alterações cognitivas em diferentes contextos de vulnerabilidade social. Rev. Gaúcha Enferm, Porto Alegre, v. 31, n. 1, p. 115-122, 2010.

SEIMA, M.D.; LENARDT, M. H.; CALDAS, C. P. Relação no cuidado entre o cuidador familiar e o idoso com Alzheimer. Rev. bras. enferm, Brasília, v.67, n. 2, p. 233-240, 2014.

VERAS, R.P.; OLIVEIRA, M. Envelhecer no Brasil: a construção de um modelo de cuidado. Ciência \& Saúde Coletiva, Rio de Janeiro, v. 23, n. 6, p. 1929-1936, 2018.

VIDIGAL, F.C. Satisfação em Cuidar de Idosos com Alzheimer: percepções dos cuidadores familiares. Cogitare Enfermagem, v.19, n.4, p.768-775, 2014.

VIEIRA, L.; NOBRE, J. R. da S.; BASTOS, C.C.B.C.; TAVARES, K. O. Cuidar de um familiar idoso dependente no domicílio: reflexões para os profissionais da saúde. Rev. bras. geriatr. gerontol, Rio de Janeiro, v.15, n. 2, p. 255-264, 2012.

WACHHOLZ, P.A.; SANTOS, R.C.C.; WOLF, L.S.P. Reconhecendo a sobrecarga e a qualidade de vida de cuidadores familiares de idosos frágeis. Rev. Bras. Geriatr. Gerontol, Rio de Janeiro, v. 16, n. 3, p. 513-526, 2013.

WATT, M.H.; PERERA, B.; ØSTBYE, T.; RANABAHU, S.; HARSHINI, R.; MASELKO, J. Caregiving expectations and challenges among elders and their adult children in Southern Sri Lanka. Ageing Soc, v. 34, n. 5, p. 838-858, 2014. 